PROCEEDINGS OF THE

AMERICAN MATHEMATICAL SOCIETY

Volume 139, Number 7, July 2011, Pages 2577-2585

S 0002-9939(2010)10690-1

Article electronically published on December 17, 2010

\title{
CIRCLE PACKING COORDINATES FOR THE MODULI SPACE OF TORI
}

\author{
G. BROCK WILLIAMS
}

(Communicated by Mario Bonk)

\begin{abstract}
We develop a set of natural coordinates $(\alpha, \beta)$ on the moduli space of Euclidean tori using the combinatorial structure of circle packings. Surfaces with rational coordinates support Brooks packings, while surfaces with rational $\alpha$ and irrational $\beta$ coordinates support generalized Brooks packings with periodic singularities.
\end{abstract}

\section{INTRODUCTION}

Paul Koebe [15, E. M. Andreev 11, and William Thurston 20, 19] independently showed that reasonable triangulations of the sphere, or equivalently, the disc, can be realized by circle packings. That is, given a combinatorial triangulation of a topological sphere or disc, there exists a geometric configuration of circles which provides an embedding of the original triangulation when the centers of tangent circles are connected by geodesics. Circle packings have since been heavily studied for their deep connections to analytic functions and applied to problems ranging from brain mapping [6, 18, to military surveillance [16].

Alan Beardon and Ken Stephenson extended Koebe's, Andreev's, and Thurston's existence result to packings on Riemann surfaces [3]. Not every compact Riemann surface supports a packing, but Phil Bowers and Ken Stephenson showed that the packable surfaces are dense in moduli space [7, 8, 4, 5].

A significant open question has been to connect the conformal structure of these surfaces with the combinatorial structure of the packings they support [18, 21, 2. In this paper, we describe a family of packings created from triangulations of a rectangular grid. These symmetric Brooks packings are themselves dense and their combinatorial structure provides coordinates for moduli space.

After a brief introduction to the moduli space of tori in section 2 and an overview of circle packings in section [3, we describe how these packings provide natural coordinates for moduli space in section 4 .

\section{THE MODUli SPACE OF TORI}

Euclidean tori are of the form $\mathbb{C} / \Gamma$, where $\Gamma$ is a discrete group which we may assume without loss of generality to be generated by the translations $z+1$ and

Received by the editors January 18, 2010 and, in revised form, July 3, 2010.

2010 Mathematics Subject Classification. Primary 52C26, 30F60.

Key words and phrases. Circle packing, moduli space.

(C)2010 American Mathematical Society

Reverts to public domain 28 years from publication 


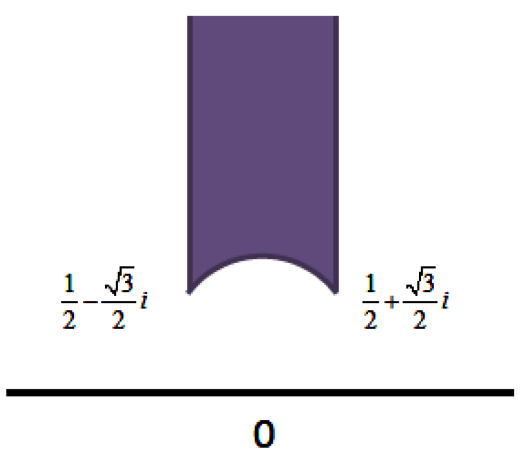

Figure 1. A fundamental region for $\mathrm{PSL}_{2}(\mathbb{Z})$. All tori are conformally equivalent to $R_{\tau}$ for some $\tau$ in this region.

$z+\tau$, with $\tau$ in the upper half plane $\mathbb{H}[13$, 14. Conversely, each $\tau \in \mathbb{H}$ determines a torus $R_{\tau}$. Two tori $R_{\tau_{1}}$ and $R_{\tau_{2}}$ are conformally equivalent iff

$$
\tau_{2}=\frac{a \tau_{1}+b}{c \tau_{1}+d},
$$

for $a, b, c, d \in \mathbb{Z}$ and $a d-b c=1$, that is, if $\tau_{1}$ and $\tau_{2}$ differ by an element of the Möbius group $\mathrm{PSL}_{2}(\mathbb{Z})$.

The moduli space $M_{1}$ of conformal equivalence classes of tori is given by $\mathbb{H} / \mathrm{PSL}_{2}(\mathbb{Z})$. A fundamental region for $M_{1}$ is the region above the unit circle and bounded by the vertical lines $x= \pm \frac{1}{2}$. See Figure 1 .

\section{Circle Packings}

3.1. Definitions and examples. Since William Thurston pointed out the connection between circle packings and analytic functions 20, 19, packings have been widely studied.

Definition 3.1. A circle packing is a locally finite configuration of circles with a specified pattern of tangencies. In particular, if $\mathcal{K}$ is a triangulation of an orientable topological surface, then a circle packing $P$ for $\mathcal{K}$ is a configuration of circles such that

(1) $P$ contains a circle $C_{v}$ for each vertex $v$ in $\mathcal{K}$,

(2) $C_{v}$ is externally tangent to $C_{u}$ if $[v, u]$ is an edge of $\mathcal{K}$,

(3) $\left\langle C_{v}, C_{u}, C_{w}\right\rangle$ forms a positively oriented mutually tangent triple of circles if $\langle v, u, w\rangle$ is a positively oriented face of $\mathcal{K}$,

(4) $P$ is locally finite; that is, any compact subset of the ambient space intersects at most finitely many circles of $P$.

A packing is called univalent if none of its circles overlap, that is, if no pair of circles intersect in more than one point.

A univalent circle packing produces a geometric realization of its underlying triangulation. Vertices can be embedded as centers of their corresponding circles, and edges can be realized as geodesic segments joining centers of circles. The collection of triangles embedded in this way is called the carrier of the packing, written carr $P$. See Figure 2 

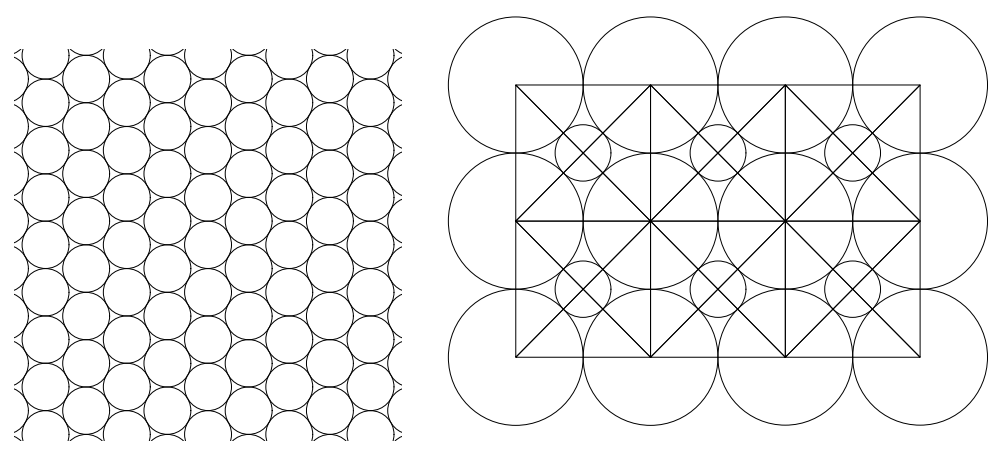

Figure 2. Portions of the regular hex packing (left) in which every circle has degree 6 and the "ball-bearing packing"(right), named by Tomasz Dubejko and Ken Stephenson [11, with its carrier illustrating its underlying grid structure.
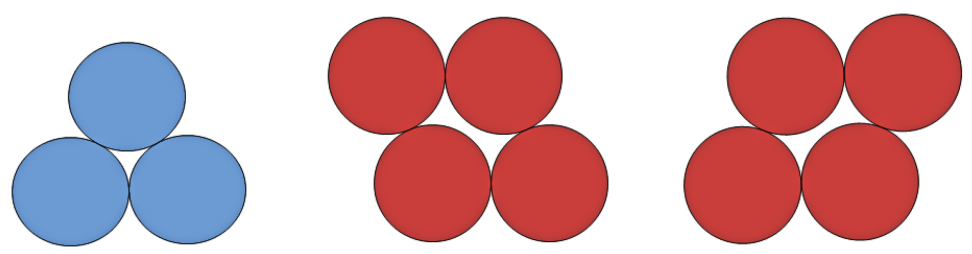

Figure 3. Three circles form a rigid structure, while four can be moved, distorting the quadrilateral interstice between them.

3.2. Brooks parameterization of quadrilateral interstices. The requirement that our circles fit together according to the dictates of an underlying triangulation is necessary to give uniqueness to the structure. A minute's experimentation with 4 coins illustrates nicely how 3 circles will "stick" together with a fixed triangular interstice between, but 4 coins bounding a quadrilateral interstice can still be moved. See Figure 3 ,

In his work on Schottky groups, Robert Brooks provided an extremely useful parameterization of these quadrilateral interstices [7, 8, 9. Label two of the nontangent bounding circles as the "top" and "bottom" and the other two circles as the "left" and "right" sides. Add a very small circle tangent to the top and left circles, then increase its radius until it hits either the bottom or the right circle. Notice that one can always add a circle that hits at least 3 of the 4 sides. Label it "horizontal" if it hits the top and bottom and "vertical" if it hits the left and right sides. Notice that in general a quadrilateral interstice will remain and the original labeling will extend in an obvious way to the new interstice, and so we may continue adding circles. See Figure 4.

Let $h_{1}$ be the number of horizontal circles which can be added before a vertical circle must be added. Let $v_{1}$ be the number of vertical circles which can then be added before requiring a horizontal circle. Repeat this procedure to define $h_{2}, h_{3}, \ldots$ and $v_{2}, v_{3}, \ldots$. Notice that if it is ever possible to add a circle which hits all four remaining sides, then only triangular interstices will remain and both $h_{n}$ and $v_{n}$ will be zero after that stage. 


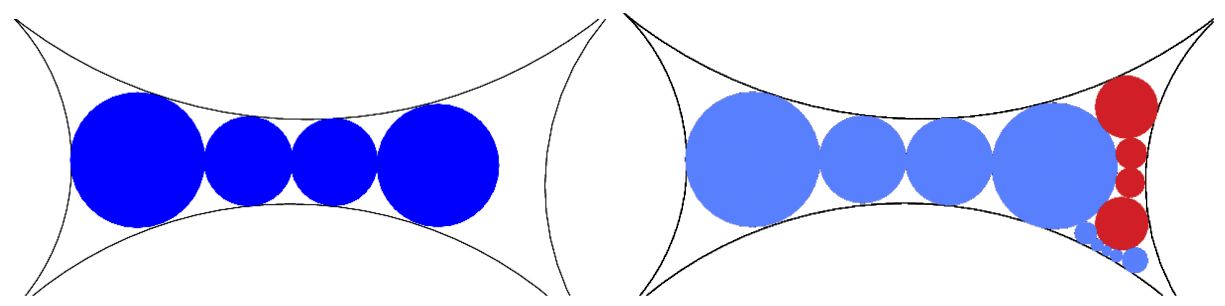

Figure 4. A quadrilateral interstice after the first (left) and third (right) stages of computing the Brooks parameter. Four horizontal circles were added, followed by 4 vertical circles, then 5 more horizontal circles.

The Brooks parameter is then defined as the continued fraction

$$
\beta=h_{1}+\frac{1}{v_{1}+\frac{1}{h_{2}+\frac{1}{v_{2}+\cdots}}} .
$$

The Brooks parameter will be rational if and only if the $h_{n}$ 's and $v_{n}$ 's eventually become zero. That is, the parameter is rational if and only if the procedure described above results in a circle packing inside the interstice. The infinite collection of circles encoded by an irrational parameter will accumulate on the bottom-right side of the interstice and thus cannot fulfill the local finiteness required in our definition of a circle packing. Brooks proved that the parameter depends continuously on the original four circles which form the quadrilateral interstice [8, 9]. The Brooks parameter thus provides a combinatorial analog of the conformal module of the interstice.

On the other hand, a positive number $\beta$ can be uniquely decomposed into a continued fraction of the form (3.1). If $\beta \in \mathbb{Q}$, this continued fraction encodes a (locally finite) triangulation $\mathcal{K}(\beta)$ of a rectangle which can be realized by a circle packing $P(\beta)$. If $\beta \notin \mathbb{Q}$, it also encodes a triangulation $\mathcal{K}(\beta)$ with a singularity at the bottom-right corner at which it is not locally finite. Since $\beta$ and the radii of the four defining circles depend continuously on one another, $\mathcal{K}(\beta)$ can be realized by a configuration $P(\beta)$ of circles which is not locally finite at the one singularity. We will follow the current literature and reserve the term "circle packing" for locally finite configurations and refer to such a $P(\beta)$ as a generalized packing with a singularity.

3.3. Packings on surfaces. Alan Beardon and Ken Stephenson showed that triangulations of surfaces can be realized by packings 3 .

Discrete Uniformization Theorem. For every (locally finite) triangulation $\mathcal{K}$ of an orientable surface, there is a unique Riemann surface $S_{\mathcal{K}}$ which supports a circle packing filling $S_{\mathcal{K}}$ and having $\mathcal{K}$ as its underlying triangulation.

The converse of the Discrete Uniformization Theorem is false for compact surfaces of genus $g>0$ as there are only countably many combinatorially distinct triangulations for each $g$, but the moduli space $M_{g}$ is uncountable. However Bowers and Stephenson showed that the packable surfaces are dense in $M_{g}$ [4, 5].

Density Theorem. For each fixed genus $g>0$, the surfaces supporting a circle packing are dense in the moduli space $M_{g}$ of compact surfaces of genus $g$. 
Bowers and Stephenson's proof is essentially the following: Take an arbitrary compact surface of genus $g>0$ and begin to cover it with circles. Circles can always be added until only triangular or quadrilateral interstices remain. Since the Brooks parameter varies continuously, the parameters of all the quadrilateral interstices can be made rational by an arbitrarily small change in the circles forming each interstice or, equivalently, by an arbitrarily small change in the conformal structure of the surface. Consequently there is a packable surface arbitrarily near the original.

\section{Packing Coordinates}

4.1. Brooks packings. Consider the graph $G$ formed by an infinite two-dimensional simply connected rectangular grid and designate some vertex of $G$ as $v_{0}$ and one of its neighbors as $v_{1}$. $G$ can be embedded in the plane as a square grid formed by line segments connecting the Gaussian integers and the vertices $v_{0}$ and $v_{1}$ embedded at the points 0 and 1 , respectively. While we wish to continue treating $G$ as a purely combinatorial graph, we will nonetheless borrow from this embedding the notions of "horizontal" and "vertical" directions in the grid in the obvious way.

If $\beta>0$, then as described in section 3.2. $\beta$ encodes a triangulation (possibly with a singularity) of a rectangle. Let $\mathcal{K}(\beta)$ be the graph resulting from triangulating each rectangle of $G$ as specified by $\beta$, being careful to consistently apply the same notion of "top," "bottom," "left," and "right" throughout the grid so that the grid remains combinatorially invariant under "horizontal" and "vertical" translations. Notice that the Brooks procedure does not add any vertices to existing edges of $G$, so $\mathcal{K}(\beta)$ is a triangulation.

Definition 4.1. A circle packing on a surface $S$ is called a Brooks packing if its underlying triangulation is formed from a rectangular grid, each rectangle of which is triangulated according the the same rational Brooks parameter $\beta$. Similarly, a configuration of circles created using an irrational Brooks parameter produces a generalized Brooks packing with singularities.

Lemma 4.2. Let $\mathcal{K}(\beta)$ be the triangulation formed from triangulating each rectangle of the infinite simply connected grid using the Brooks parameter $\beta$ as described above. Then there exists a Brooks packing $P(\beta)$ (or generalized Brooks packing with singularities if $\beta \notin \mathbb{Q})$ for $\mathcal{K}(\beta)$ filling $\mathbb{C}$ and unique up to conformal automorphisms of $\mathbb{C}$. In particular, we can take the circle $C_{0}$ corresponding to $v_{0}$ and the circle $C_{1}$ corresponding to $v_{1}$ to be centered at the points 0 and 1 , respectively.

Proof. If $\beta \in \mathbb{Q}$, then $\mathcal{K}(\beta)$ is an infinite simply connected locally finite triangulation. The Discrete Uniformization Theorem thus implies the existence of a circle packing $P(\beta)$ filling either $\mathbb{D}$ or $\mathbb{C}$ and unique up to conformal automorphisms of the ambient space. Suppose the packing fills $\mathbb{D}$. Notice that $K(\beta)$ has a $\mathbb{Z} \times \mathbb{Z}$ symmetry group, which implies the existence of a subgroup of $P S L_{2}(\mathbb{R})$, the automorphism group of $\mathbb{D}$, which is isomorphic to $\mathbb{Z} \times \mathbb{Z}$. Since no such subgroup exists, $P(\beta)$ must fill $\mathbb{C}$. One can also show that $P(\beta)$ must fill $\mathbb{C}$ by applying the random walk criteria of Zheng-Xu He and Oded Schramm [12, the electrical reasoning of Peter Doyle and J. Laurie Snell [10, and the recurrence/transience results of George Pólya [17.

Suppose $\beta \notin \mathbb{Q}$. Since $\beta$ and the radii of the four defining circles depend continuously on one another, if we take a sequence of rational numbers converging to $\beta$, the radii of the circles corresponding to vertices of the rectangular grid must 


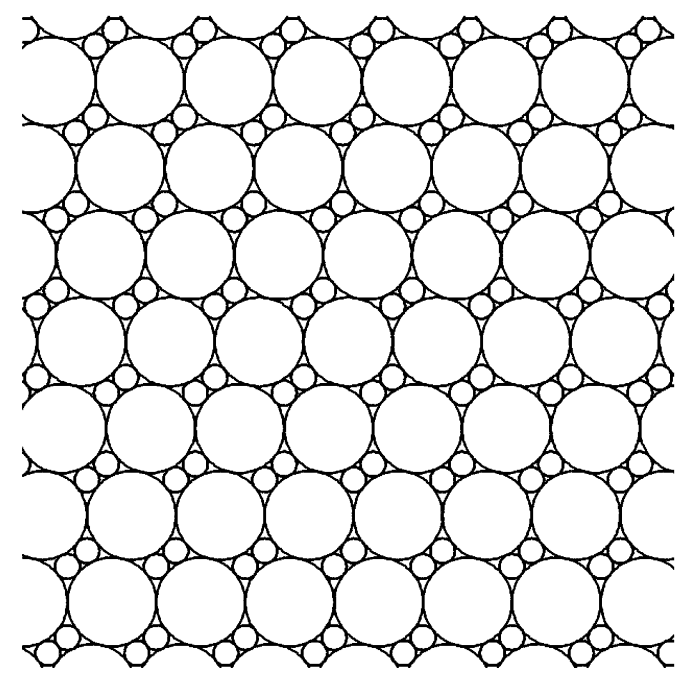

Figure 5 . The infinite packing $P(2)$. Notice that the $\mathbb{Z} \times \mathbb{Z}$ symmetry group forces all the large circles corresponding to vertices in the original grid to have the same radii.

converge to radii for circles whose quadrilateral interstices have Brooks parameter $\beta$. These interstices can clearly then be filled with circles in the pattern specified by $\beta$ to produce the generalized packing with singularities $P(\beta)$.

The uniqueness of $P(\beta)$ for rational $\beta$ and the continuous dependence of the circles on $\beta$ imply that the same uniqueness of $P(\beta)$ must hold for irrational $\beta$.

In the notation of Lemma 4.2, $P(1)$ is simply the ball bearing packing of Figure 2 , Notice that in Figures 2 and 5, the large circles corresponding to vertices of the original grid all have the same radius. The symmetry of $\mathcal{K}(\beta)$ and the uniqueness of $P(\beta)$ imply that the same must be true for all $\beta>0$.

Lemma 4.3. If $P(\beta)$ is the normalized Brooks packing (or generalized Brooks packing with singularities) of Lemma 4.2, all circles $C_{v}$ corresponding to vertices $v$ of the underlying grid must have the same radius.

Proof. Suppose some circle $C_{v}$ had a different radius than the circle $C_{v_{0}}$ which we normalized to be centered at 0 . Because $\mathcal{K}(\beta)$ is combinatorially invariant under "vertical" and "horizontal" translations of the original infinite grid, there is a neighbor $u$ of $v$ and a graph automorphism $\gamma$ of $\mathcal{K}(\beta)$ sending $v$ to $v_{0}$ and $u$ to $v_{1}$.

Now apply a conformal automorphism $g$ to $P(\beta)$ to translate $C_{v}$ to 0 and $C_{u}$ to 1 , producing a new renormalized packing $g(P(\beta))$ for the triangulation $\gamma(\mathcal{K}(\beta))$. But the infinite triangulations $\gamma(\mathcal{K}(\beta))$ and $\mathcal{K}(\beta)$ are combinatorially indistinguishable; thus $g(P(\beta))$ is a packing for $\mathcal{K}(\beta)$ itself. By the uniqueness part of Lemma 4.2, the radii of $C_{v}$ and $C_{v_{0}}$ must be the same.

4.2. Coordinates. Let $\mathcal{K}_{m, n}(\beta)$ be the $m \times n$ rectangular piece of the triangulated grid $\mathcal{K}(\beta)$ with $v_{0}$ as its lower left vertex. Consider the portion of $P(\beta)$ corresponding to $\mathcal{K}_{m, n}$. Scale this packing by $1 / n$ so that $C_{0}$ remains centered at 0 , but 


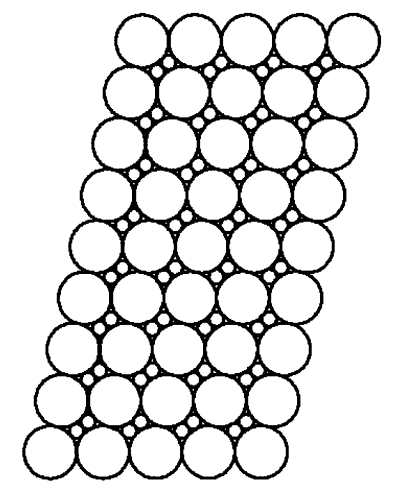

Figure 6. The packing $P_{8,4}(2)$, which lifts to a Brooks packing on the torus $R_{\tau(8 / 4,2)}$.

the circle in the lower right corner is centered at 1 . Call this packing $P_{m, n}$ and let $\tau(m / n, \beta)$ denote the center of the circle at the upper left corner of the packing. By our normalization and the symmetry of $P(\beta)$, each of circles in $P_{m, n}(\beta)$ corresponding to vertices of the original grid has radius $1 /(2 n)$. Because of our renormalization and the symmetry of $P(\beta), \tau(m / n, \beta)$ is well-defined.

By Lemma 4.3. $P_{m, n}(\beta)$ lifts to a Brooks packing on the torus $R_{\tau(m / n, \beta)}$. See Figure 6.

Moreover, $|\tau(m / n, \beta)|=m / n$ since the line from 0 to $\tau(m / n, \beta)$ is comprised of $2 m$ radii each of length $1 /(2 n)$. Thus as $\beta$ varies, $\tau(m / n, \beta)$ sweeps out an arc of a circle centered at 0 of radius $m / n$. Notice that the argument $\theta$ of $\tau(m / n, \beta)$ depends only on $\beta$ and there is a one-to-one correspondence $f(\beta)$ between $\beta$ and $\theta$.

Notice that when $\beta=1, P_{m, n}(\beta)$ is a portion of the ball-bearing packing and the edges of the underlying original form squares in carr $P_{m, n}(\beta)$. Thus $\tau(m / n, 1)=$ $(m / n) i$.

As $\beta \rightarrow \infty$, the packings "slide to the right"; that is, the top and bottom circle of each quadrilateral interstice move closer together. In the limit, each circle will have 6 neighbors, producing the regular hex packing. Similarly, and as $\beta \rightarrow 0$, the packings "slide to the left," and the left and right circles approach one another. The limit is again the regular hex packing. Thus as $\beta$ varies from 0 to infinity, $\tau(m / n, \beta)$ sweeps out an arc of measure $\pi / 3$ from $(m / n) e^{i 2 \pi / 3}$ to $(m / n) e^{i \pi / 3}$.

We can extend the definition of $\tau(m / n, \beta)$ to $\tau(\alpha, \beta)$ for positive real numbers $\alpha \geq 1$ by continuity. Notice that for $\alpha \geq 1$, and $0<\beta<\infty$, these arcs cover the fundamental region for $\mathrm{PSL}_{2} \mathbb{Z}$ pictured in Figure 1. Consequently, we have the following theorem:

Theorem 4.4. The construction described above provides coordinates $(\alpha, \beta)$ for the moduli space $M_{1}$ of tori. Moreover, $R_{\tau}$ supports a Brooks packing if $\tau=\tau(\alpha, \beta)$ for $\alpha, \beta \in \mathbb{Q}$ and a generalized Brooks packing with periodic singularities if $\alpha \in \mathbb{Q}$ but $\beta \notin \mathbb{Q}$.

As an immediate corollary, we also see that not only are the packable tori dense in the moduli space [4, 5], but the tori which support Brooks packings in particular are dense. 
4.3. Observations about the correspondence. We would like to thank the referee for pointing out the following observations about the correspondence $f(\beta)$ between Brooks's parameters and angles $\theta=\operatorname{Arg}(\tau)$ :

- $f(\beta)$ is decreasing

- $f(\beta)+f\left(\frac{1}{\beta}\right)=\pi$

- $f(0)=\frac{2 \pi}{3}$

- $f(1)=\frac{\pi}{2}$

- $f(2)=\cos ^{-1}\left(\frac{5-\sqrt{17}}{4}\right)$

- $f(3)=2 \sin ^{-1}(x)$, where $x \in(0,1)$ satisfies $x^{4}+x^{3}+x=1 / 2$

- $f(\infty)=\frac{\pi}{3}$

- In view of Brooks's non-differentiability result [9], it is reasonable to conjecture that $f$ is not differentiable at rational values of $\beta$.

\section{REFERENCES}

1. E. M. Andreev, Convex polyhedra in Lobacevskii space, Mat. Sb. (N.S.) 10 (1970), 413-440 (English). MR0259734(41:4367)

2. R.W. Barnard and G. Brock Williams, Combinatorial excursions in moduli space, Pacific J. Math. 205 (2002), no. 1, 3-30. MR1921075 (2003f:52017)

3. Alan F. Beardon and Kenneth Stephenson, The uniformization theorem for circle packings, Indiana Univ. Math. J. 39 (1990), 1383-1425. MR1087197(92b:52038)

4. Philip L. Bowers and Kenneth Stephenson, The set of circle packing points in the Teichmüller space of a surface of finite conformal type is dense, Math. Proc. Camb. Phil. Soc. 111 (1992), 487-513. MR1151326 (93a:30050)

5. _ Circle packings in surfaces of finite type: An in situ approach with application to moduli, Topology 32 (1993), 157-183. MR1204413 (94d:30083)

6. Phillip L. Bowers and Monica K. Hurdal et al., Quasi-conformally flat mapping the human cerebellum, Medical Image Computing and Computer-Assisted Intervention (C. Taylor and A. Colchester, eds.), Lecture Notes in Computer Science, vol. 1679, Springer, 1999, pp. 279286.

7. Robert Brooks, On the deformation theory of classical Schottky groups, Duke Math. J. 52 (1985), 1009-1024. MR816397 (87g:32024)

8. _ Circle packings and co-compact extensions of Kleinian groups, Inventiones Mathematicae 86 (1986), 461-469. MR860677 (88b:32050)

9. - The continued fraction parameter in the deformation theory of classical Schottky groups, Contemp. Math., vol. 136, Amer. Math. Soc., Providence, RI, 1992, pp. 41-54. MR.1188193 (93j:52029)

10. Peter G. Doyle and J. Laurie Snell, Random walks and electrical networks, MAA, 1984. MR 920811 (89a:94023)

11. Tomasz Dubejko and Kenneth Stephenson, Circle packing: Experiments in discrete analytic function theory, Experiment. Math. 4 (1995), no. 4, 307-348. MR1387696 (97f:52027)

12. Zheng-Xu He and Oded Schramm, Hyperbolic and parabolic packings, Discrete \& Computational Geom. 14 (1995), 123-149. MR1331923 (96h:52017)

13. Yoichi Imayoshi and Masahiko Taniguchi, An introduction to Teichmüller spaces, SpringerVerlag, 1992. MR.1215481 (94b:32031)

14. Gareth Jones and David Singerman, Complex functions: An algebraic and geometric viewpoint, Cambridge University Press, Cambridge, 1987. MR890746 (89b:30001)

15. Paul Koebe, Kontaktprobleme der Konformen Abbildung, Ber. Sächs. Akad. Wiss. Leipzig, Math.-Phys. Kl. 88 (1936), 141-164.

16. Eric M. Murphy and Michael D. Payne, Coverage, control, and reconnaissance: A new perspective on surveillance and reconnaissance sufficiency analysis, preprint.

17. G. Pólya, Über eine Aufgabe der Wahrscheinlichkeitsrechnung betreffend die Irrfahrt im Strassennetz, Math. Ann. 84 (1921), 149-160. MR.1512028 
18. Kenneth Stephenson, Introduction to circle packing, Cambridge University Press, Cambridge, 2005, The theory of discrete analytic functions. MR2131318

19. William Thurston, The geometry and topology of 3-manifolds, Princeton University Notes, preprint.

20. - The finite Riemann mapping theorem, 1985, Invited talk, An International Symposium at Purdue University on the occasion of the proof of the Bieberbach conjecture, March 1985.

21. G. Brock Williams, Earthquakes and circle packings, J. Anal. Math. (2001), no. 85, 371-396. MR:1869616 (2003e:57032)

Department of Mathematics, Texas Tech University, Lubbock, Texas 79409

E-mail address: brock.williams@ttu.edu

URL: http://www.math.ttu.edu/ williams 